\title{
Compact Duplexing for a 680-GHz Radar Using a Waveguide Orthomode Transducer
}

\author{
Carlos A. Leal-Sevillano, \\ Emmanuel Decrossas, Robert J. Dengler, \\ José R. Montejo-Garai, Goutam Chattopadhyay,
}

Ken B. Cooper,

Jorge A. Ruiz-Cruz,

and Jesús M. Rebollar

\begin{abstract}
A compact $680-\mathrm{GHz}$ waveguide orthomode transducer (OMT) and circular horn combination has been designed, tested, and characterized in a radar transceiver's duplexer. The duplexing capability is implemented by a hybrid waveguide quasi optical solution, combining a linear polarization OMT and an external grating polarizer. Isolation between the OMT's orthogonal ports' flanges was measured with a vector network analyzer to exceed $33 \mathrm{~dB}$ over a $>10 \%$ bandwidth between 630 and $710 \mathrm{GHz}$. Calibrated Y-factor measurements using a mixer attached to the OMT ports reveal losses through the transmit and receive paths that sum to an average of $4.7 \mathrm{~dB}$ of two-way loss over $660-690 \mathrm{GHz}$. This is consistent with radar sensitivity measurements comparing the new OMT/horn with a quasi-optical wire grid beam splitter. Moreover; the radar performance assessment validates the OMT as a suitable compact substitute of the wire grid for the JPL's short-range 680-GHz imaging radar.
\end{abstract}

Index Terms - Duplexer, orthomode transducer, terahertz radar.

\section{INTRODUCTION}

M ONOSTATIC continuous-wave radar transceivers must include a duplexer that couples the transmit port to outgoing radiation and the receive port to incoming radiation, while simultaneously isolating the receiver from the transmitter to prevent interference or noise leakage. In the submillimeter-wave regime, as a rule, solid-state radars operate in a frequency-modulated continuous-wave (FMCW) mode because low available powers preclude pulsed operation. Furthermore, because phase-noise levels worsen rapidly with increasing frequency, high transmit/receive (T/R) isolation is especially important in the terahertz $(\mathrm{THz})$ regime. Circulators
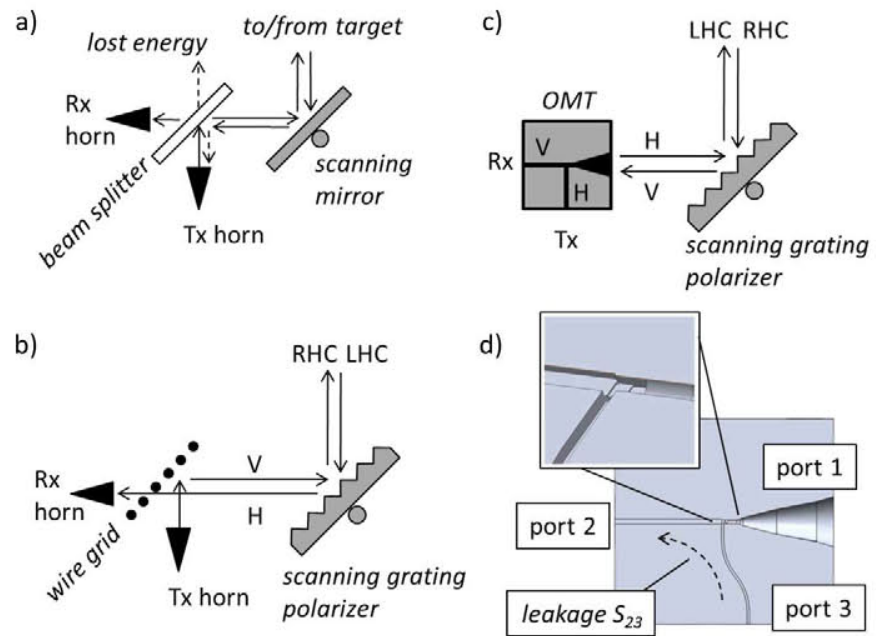

Fig. 1. THz radar duplexing alternatives. (a) Two horns and a beam splitter (b) Two horns, a wire grid, and a grating polarizer. (c) Hybrid waveguide quasioptical duplexing solution. (d) Schematic of the linear polarization OMT/horn.

are the most common duplexing components at lower frequencies, but, above $100 \mathrm{GHz}$ low-loss circulators are not available or not practical. An alternative simple duplexing method is to place a $50 / 50$ beam splitter at a $45^{\circ}$ angle between orthogonally pointing transmit and receive horns [1], [2], as in Fig. 1(a). However, this approach results in a best case signal-to-noise ratio (SNR) penalty of $6 \mathrm{~dB}$ because half of the beam energy is lost for each pass of the signal.

Previously we introduced an effective method for overcoming this SNR penalty in a $680-\mathrm{GHz}$ radar by using a wire grid and grating reflector combination [Fig. 1(b)], which radiates and detects circularly polarized beams of opposite handedness with near-perfect efficiency and excellent transmit/receive (T/R) isolation exceeding $90 \mathrm{~dB}$ [3]. While this all-quasi-optical grid/grating duplexer overcomes most of the 6-dB beam splitter loss, it still requires a polarizing grid that, like the beam splitter, is delicate and difficult to integrate with a densely spaced focal plane array of transceivers. The development of such arrays is a long-term goal for many radar development efforts in the millimeter- and submillimeter-wave regimes because of the potential for high-speed image acquisition or phased-array antennas.

To overcome this limitation to achieving the densest possible packaging, we have designed and tested a compact waveguide orthomode transducer (OMT), including an integrated horn, that substitutes for the wire grid in a $680-\mathrm{GHz}$ duplexer [Fig. 1(c)]. 
This is similar in spirit to the septum-OMT we previously built for a 225-GHz radar [4], but, by relying on an external grating polarizer instead of a waveguide septum geometry to generate circular polarization, the transmit and receive ports are orthogonally polarized with respect to each other to achieve the highest possible isolation [Fig. 1(d)]. With a modest bandwidth requirement of 5\%, the new OMT design was made with onefold symmetry with a lateral coupled port, trading off bandwidth in favor of fabrication simplicity and, therefore, high isolation. For similar reasons, a smooth-walled, multiflare-angled circular horn antenna was chosen for the OMT's common port. Our design is well suited for use in focal plane arrays because it is compatible with low-cost microfabrication techniques, and it requires virtually no more volume than a single 680 -GHz horn antenna.

\section{OMT AND HORN DESIGN}

\section{A. Geometry Selection}

An OMT is a passive device with three physical ports that combine/divide two orthogonal linear polarizations at a common port. OMTs are key elements in many antenna feed configurations ranging from microwave satellite communication equipment to millimeter-wave radioastronomy receivers. When reduced mass and volume are a priority, waveguide-based devices, as opposed to quasi-optical geometries using wire grids or other free-space structures, are favored for very high-frequency applications.

There are many possible waveguide OMT geometries providing different electric characteristics, namely bandwidth, isolation, and cross polarization coupling. A survey of the most common used OMT structures can be found in [5] and [6].

High-isolation OMT junctions can be gathered in two categories: onefold symmetry or twofold symmetry. The main difference relies upon the maximum achievable bandwidth. While twofold symmetric OMTs offer wide bandwidth, their main drawback is that they are substantially more complex and difficult to fabricate at high frequency.

Based on this and taking into account the $680-\mathrm{GHz}$ radar's system specifications of $5 \%$ bandwidth $(660-690 \mathrm{GHz})$ with isolation as high as possible, as well as the fabrication limitations ( $\sim 3-\mu \mathrm{m}$ waveguide dimension tolerance) for the metal block, a onefold symmetric OMT design was chosen.

The most common asymmetric OMT uses a septum and a thin iris [7]-[10] to provide the required polarization separation. However, the septum structure is very difficult to fabricate for 680-GHz operation, and the approaches of [11] and [12] are more appropriate for our situation. These approaches also avoid the use of other critical elements, such as ridges, pines, or small features. A modification of the latter approach operating at $600 \mathrm{GHz}$ has been recently presented in [13] with successful results.

The OMT design we propose in this paper is based on extracting the horizontal polarization through the lateral port (according to the definition in Fig. 2) using a T-shaped waveguide. The vertical polarization is guided to the axial port via a waveguide transformer. This geometry is inspired by the designs presented in [12] and [13], but with two significant modifications. First, to facilitate the OMT's fabrication using an end-mill, the

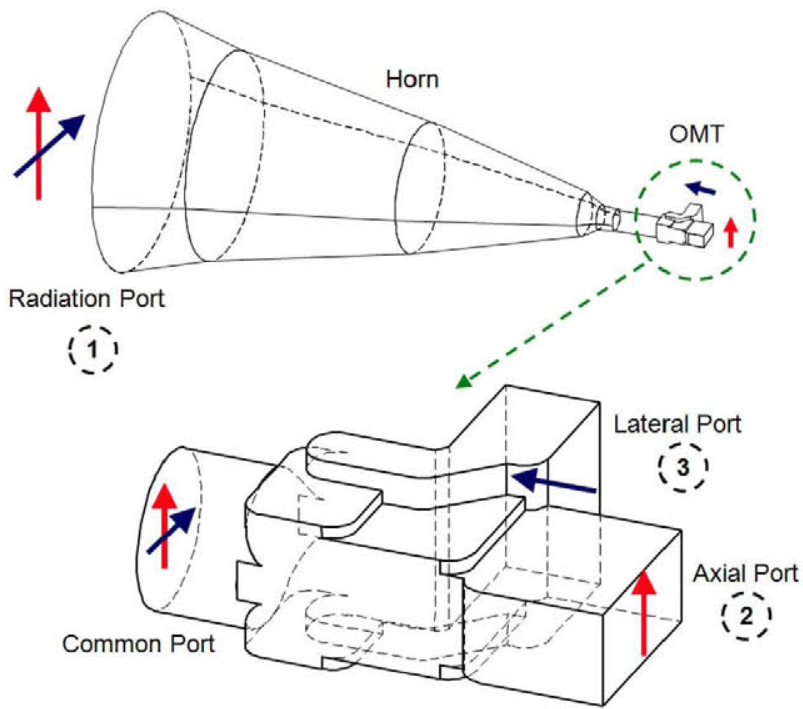

Fig. 2. Geometry of the designed OMT and horn antenna.

number of waveguide steps was reduced to only two for the axial port, along with a single finger-shape step to guide the horizontal polarization through the lateral port. Second, a square-to-circular waveguide transition was included in the design in order to transition to a horn, leading to a simple structure with a direct flush connection between square and circular waveguide, as shown in Fig. 2.

The OMT is typically followed at the common port by a circular horn antenna, such as corrugated- or smooth-profiled horn antennas [14]. In general, corrugated horn antennas give superior performance, mainly in terms of bandwidth and cross polarization. However, their fabrication is complicated, especially at high frequencies. For the radar system here, where a bandwidth less than $10 \%$ is required, a smooth-profiled hom is sufficient and can be integrated together with the OMT in a single split-block.

\section{B. Full-Wave Design and Optimization}

The horn antenna was designed using an efficient custom mode-matching method with the following procedure. First, the smooth horn profile was discretized in a finite number of steps. Then, generalized scattering matrix (GSM) of each step is calculated and cascaded as explained in [4] and [14]. Next, the profile of the horn was optimized to obtain a symmetric radiation pattern and a gain of around $25 \mathrm{dBi}$. The maximum cross polarization of the horn is not a very critical parameter for the radar application investigated here, but its value has been maintained below $-23 \mathrm{~dB}$ in $>10 \%$ bandwidth. In Fig. 3 , the computed radiation pattern at the center frequency is shown. The reflection coefficient was maintained below $-30 \mathrm{~dB}$ in the operating band, as shown in Fig. 4. It should be noted that the reflection coefficients of the $T E_{11 \mathrm{C}}$ and $T E_{11 S}$ modes are the same due to the symmetry of revolution of the horn. In addition, both modes are ideally completely decoupled.

The full-wave analysis of the OMT was carried out using CST Microwave Studio. The simulated annealing and simplex algorithms were used for the optimization of the structure. The symmetry of the OMT allowed for a shorter simulation time 


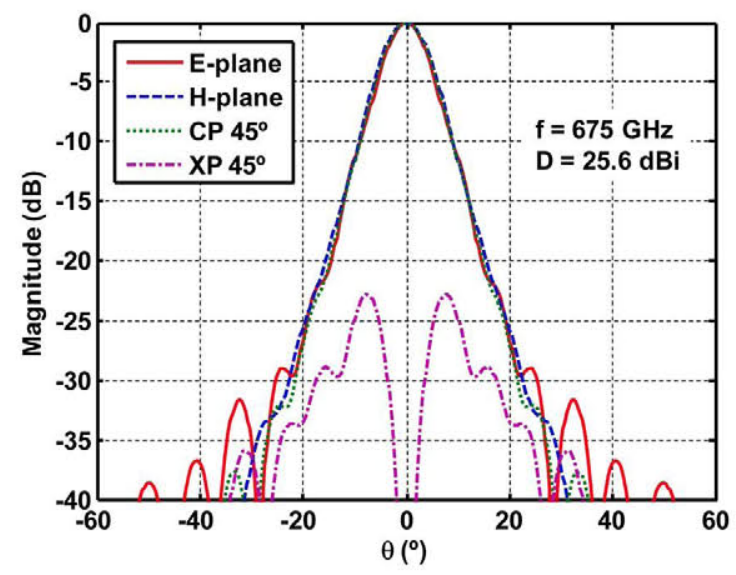

Fig. 3. Simulated radiation pattern of the horn antenna at $675 \mathrm{GHz}$.

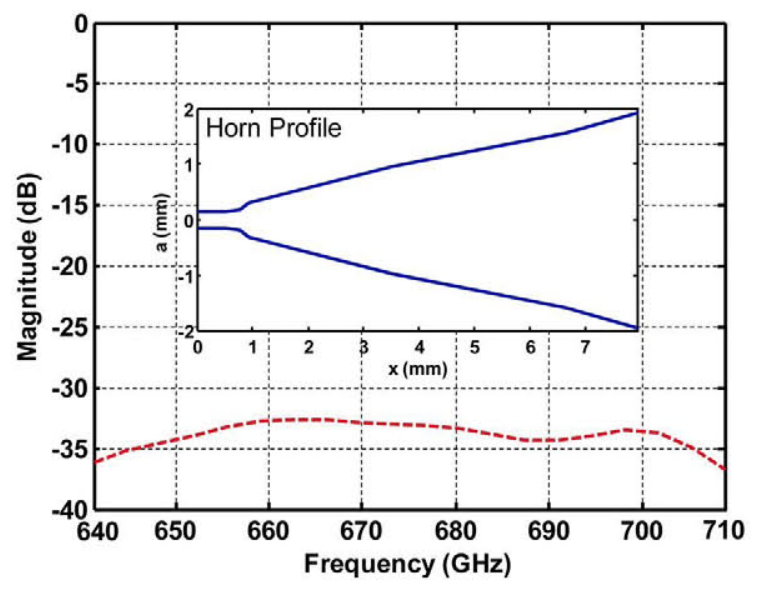

Fig. 4. Simulated reflection coefficient of the horn antenna.

with more accurate results by analyzing two electromagnetic half-problems: the OMT with a perfect electric wall (PEW) and with a perfect magnetic wall (PMW) at the symmetry plane. In addition, all of the critical dimensions of the OMT were constrained to values that can be implemented by a $3: 1$ depth/diameter ratio end-mill.

A minimum circular waveguide length between the OMT and horn was fixed in order to attenuate the higher order modes by at least $40 \mathrm{~dB}$, in particular, the $\mathbf{T M}_{01}$ generated from the lateral port of the OMT. In that way the radiation pattern of the horn antenna is not affected by the feeding network. In Fig. 5 the simulated reflection coefficients for both polarizations of the OMT and horn together are shown. The results are below $-20 \mathrm{~dB}$ in more than $10 \%$ fractional bandwidth center at $675 \mathrm{GHz}$. These levels are limited by the OMT structure, not the horn matching. The minimum return loss level as well as the bandwidth can be improved by adding more waveguide steps at the OMT, but at the cost of additional manufacturing complexity.

We note that, although the OMT was designed for machining in metal, the proposed structure is also suitable for microfabrication processes such as silicon deep-reactive ion etching [15]-[17] with minimal additional redesign. In this way, batch-processing of many OMTs simultaneously could be done, which will be more suitable for focal plane array applications.

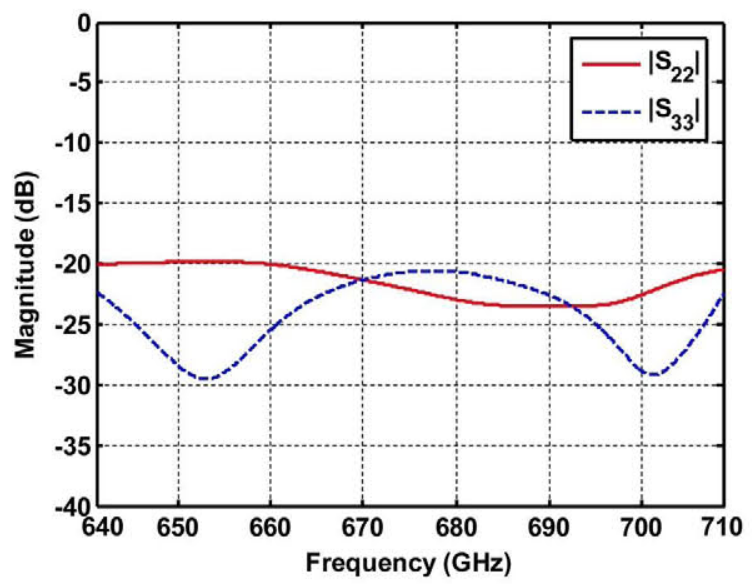

Fig. 5. Simulated reflection coefficients of the OMT and horn together for both polarizations.

\section{OMT and Horn Fabrication}

For testing purposes, a metal split-waveguide block for the OMT and horn was designed to be geometrically compatible as a drop-in component for the Jet Propulsion Laboratory's (JPL's) existing 680-GHz radar front-end module, as shown in Fig. 6. The transmit (Tx) and receive $(\mathrm{Rx})$ flanges of the OMT are on adjacent sides of the rectangular waveguide block, and their sizes constrain the minimum component dimensions, resulting in the model shown. Thus, an additional $8.3-\mathrm{mm}$ length of WR1.5 waveguide for each port links the OMT with the flanges. These waveguide lengths result in significant ohmic loss (typically tenths of a decibel per millimeter) and can be reduced in a future transceiver that is specifically designed for an OMT duplexer. The horn and OMT were manufactured in the same metal split-block, making the complete system very compact and minimizing possible alignment errors that can accumulate between multiple blocks.

The gold-plated brass OMT block was fabricated by the SESE Terahertz Lab at Arizona State University. Inspection with a microscope revealed that in the critical junction region of the OMT structure, the straight waveguide channel dimensions are within $1 \mu \mathrm{m}$ tolerance; the step lengths in the OMT are between $4-10 \mu \mathrm{m}$ too short; in the top block an unwanted recess of $28 \mu \mathrm{m}$ is present; and there is a $4-\mu \mathrm{m}$ misalignment between the top and bottom blocks; see Fig. 7. Considering the ultrasmall dimensions for $\mathrm{CNC}$ machining, this is a remarkable fabrication achievement, while still indicating that even better results may be achieved in a higher precision micromachining technique such as lithographic etching of silicon where single-micron tolerances are routine.

\section{VNA AND Y-FACTOR CHARACTERIZATION}

The three most relevant aspects of the OMT's performance for $680-\mathrm{GHz}$ imaging radar applications are insertion loss from each waveguide port to free space, isolation between the waveguide ports, and the horn antenna's co-polarization beam patterns. Other parameters that are not as important for our particular imaging radar, but may be for other applications such as communication, are return loss of the waveguide ports and 
a)

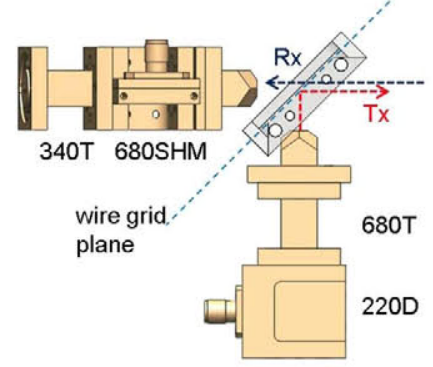

b)

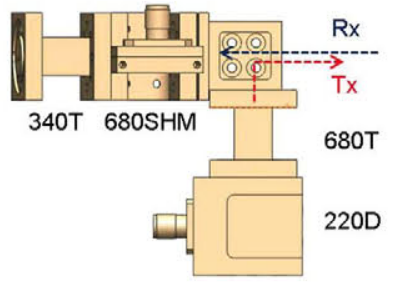

c)

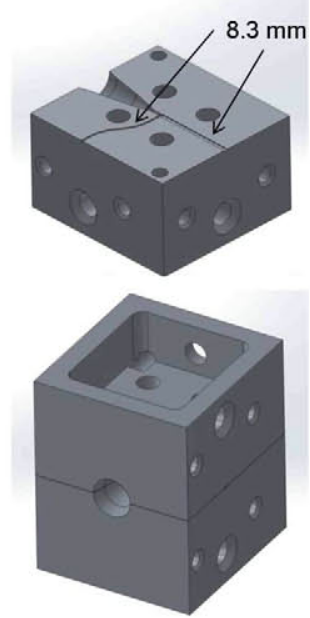

Fig. 6. Radar front-end configurations. (a) Wire grid duplexer. (b) OMT/horn block. (c) Detail of the OMT/horn split-block.
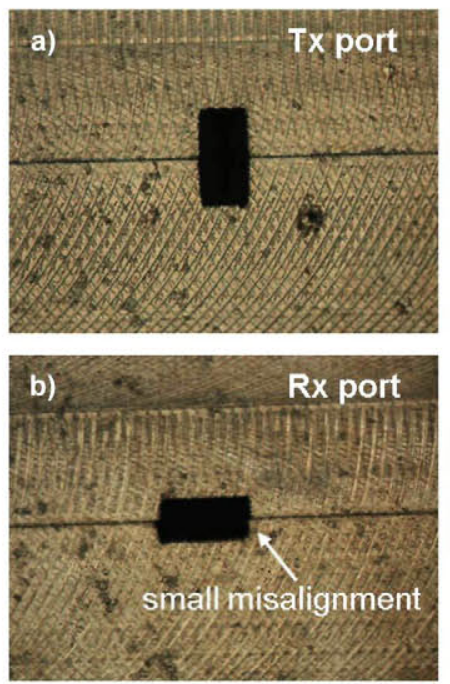
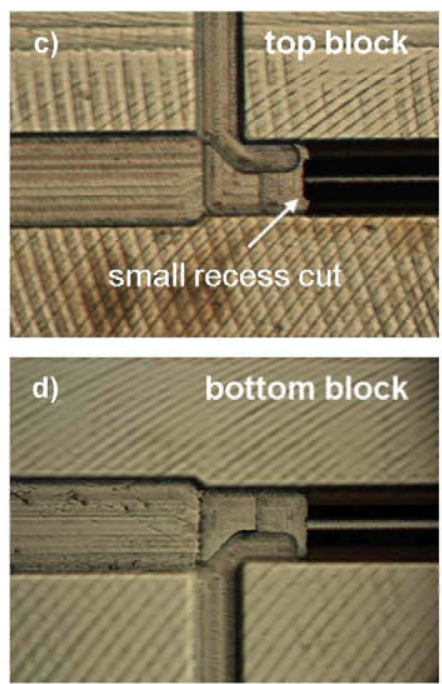

Fig. 7. Photographs of the fabricated split-block pointing out some unwanted results.

cross-polarization of the horn. Here we present measurements pertaining to insertion loss, return loss, and isolation.

Isolation and return loss measurements of the two waveguide ports were made using an Agilent N5245A Vector Network Analyzer (VNA) with WR1.5 500-750-GHz extension heads from Virginia Diodes Inc. (VDI), and through-reflect-line (TRL) calibration. These are summarized in Fig. 8. The worst-case return loss between $640-710 \mathrm{GHz}$ was $18 \mathrm{~dB}$ for Port 1 , and $>20 \mathrm{~dB}$ is attained in the radar's 660-690-GHz bandwidth. The ripples observed in the measurements correspond to standing waves between OMT and flanges. The isolation exceeds $33.5 \mathrm{~dB}$ over 640-710 GHz, and, from repeated measurements using different VNA calibrations, we estimate an error of $0.5 \mathrm{~dB}$. As a comparison, the isolation of the polarizing septum-OMT at $225 \mathrm{GHz}$ (reported in [4]) exceeded $30 \mathrm{~dB}$ over its designed bandwidth. Considering a $3 \times$ higher sensitivity to fabrication tolerances in the 680-GHz OMT, the isolation achieved here is excellent. It is

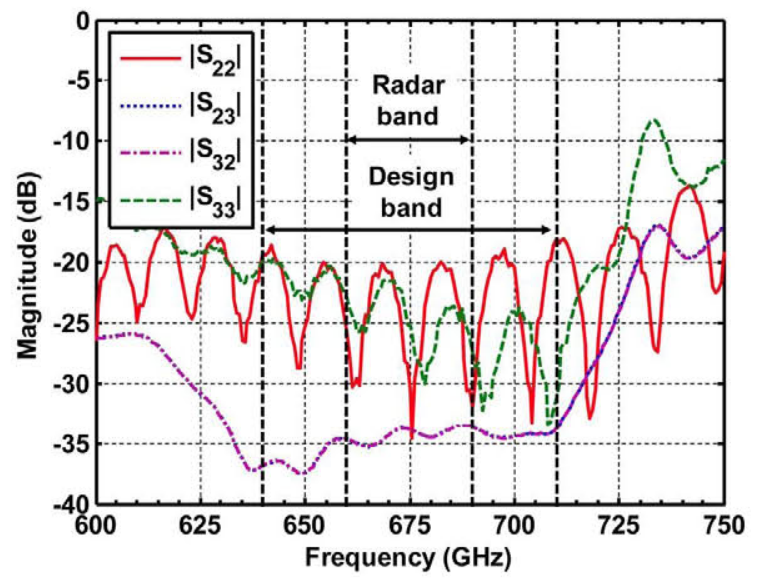

Fig. 8. Measured scattering parameters of the OMT/horn.

also much better than the $20-28$-dB isolation (depending on the port pairs) measured over $660-690 \mathrm{GHz}$ in a previously built metal waveguide hybrid coupler and described in [18]. The superior results of Fig. 8 are likely a consequence of a careful optimized OMT design with reduced number of critical features, the use of a design with better inherent isolation from using orthogonally polarized waveguide ports, the integration of the OMT and horn in the same split-block, and high-quality metal micromachining.

It is noteworthy that the high level of return loss obtained even with machining errors between $4-10 \mu \mathrm{m}$, as mentioned in Section II. This shows how a low sensitivity in the dimensions of the OMT make this topology an appropriate choice for submillimeter-wave bands. In order to further explain the measured isolation, the OMT/horn block was simulated with a misalignment of $4 \mu \mathrm{m}$ between the top and bottom halves. The comparison between simulated results and measurements are shown in Fig. 9. The simulation of the complete feed, OMT and horn, was carried out with CST Microwave Studio. Good agreement can be observed, meaning that the finite isolation measured using the VNA is consistent with a very small, yet still significant, machining error.

To measure loss through the OMT/horn, i.e., $S_{13}$ and $S_{23}$, hot/cold load Y-factor measurements of a $670 \mathrm{GHz}$ VDI subharmonic mixer were performed with its RF port connected to: 1) a standard WR1.5 VDI diagonal horn; 2) Port 1 of the OMT/horn; and 3) Port 2 of the OMT/horn. In this way, we can take ratios of measurements 1) and 2) to estimate $S_{13}$, and 1) and 3) to estimate $S_{23}$. The mixer was pumped using a LO chain with a bandwidth limited to $330-345 \mathrm{GHz}$ (for 660-690-GHz sensitivity), and the horn attached to the mixer's RF port was pointed toward echosorb loads at $77 \mathrm{~K}$ and $290 \mathrm{~K}$. The mixer's IF port was connected to a low-noise amplifier (nominal NF of $0.5 \mathrm{~dB}$ ) with baseband down-conversion to detect a $30-\mathrm{MHz}$ detection bandwidth centered at $3.6 \mathrm{GHz}$.

Fig. 10(a) shows the measured conversion loss of the mixer for each of its three RF port configurations. Fig. 10(b) shows the decibel differences of the measurements, which are estimates of the magnitudes $S_{12}$ and $S_{13}$ over the 660-690-GHz bandwidth. It is noteworthy that the insertion loss from Port 2 to free space exceeds that from Port 3, despite the equal $8.3-\mathrm{mm}$ waveguide 


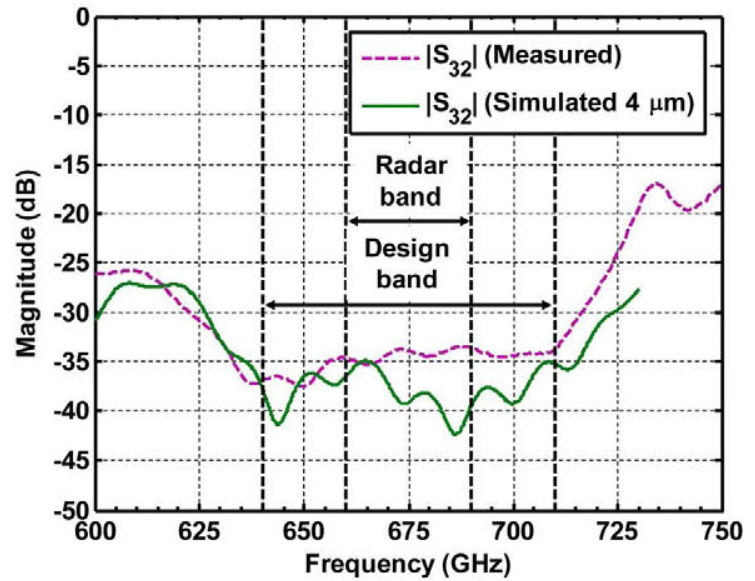

Fig. 9. Comparison between measured and simulated isolation. The simulation includes $4-\mu \mathrm{m}$ misalignment and around $0.3 \mathrm{~dB} / \mathrm{mm}$ loss at the $8.3-\mathrm{mm}$ waveguide lengths.
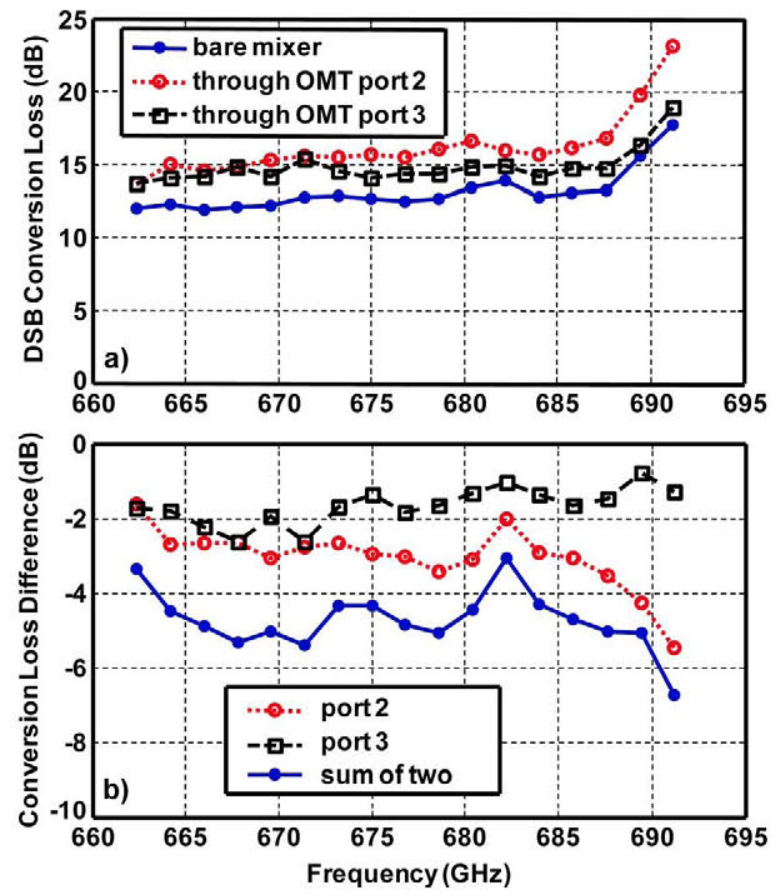

Fig. 10. Y-factor measurements. (a) Conversion loss and (b) conversion loss difference (OMT insertion loss).

lengths from the OMT to the two ports. This asymmetry in loss is likely from the Port 2 waveguide being split in its H-plane, where ohmic loss are known to exceed those in an E-plane split because the latter's modes currents vanish by symmetry across the lossy split plane interface.

In our 660-690 GHz radar system, the round-trip signal path through the OMT/horn encompasses both the $2 \rightarrow 1\left(\mathrm{~S}_{12}\right)$ and the $1 \rightarrow 3\left(\mathrm{~S}_{31}\right)$ paths, and therefore the average sum of the losses in Fig. 10(b), $4.6 \pm 0.2 \mathrm{~dB}$, is the expected level of SNR degradation due to ohmic loss through the OMT/horn. The contribution to the total level of insertion loss coming from combined horn, OMT and $8.3 \mathrm{~mm}$ waveguide lengths to the flanges can be estimated at $15 \%, 15 \%$, and $70 \%$, respectively.
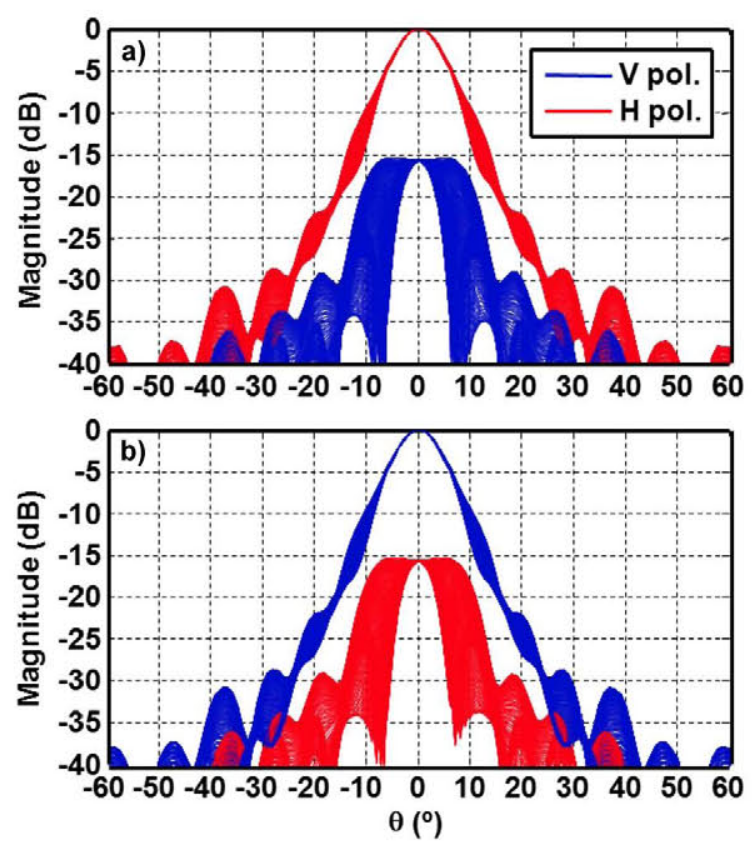

Fig. 11. Simulated radiation patterns at $675 \mathrm{GHz}$ with a misalignment of $4 \mu \mathrm{m}$ between blocks. Different azimuth cuts from 0 to $360^{\circ}$. Radiation pattern when excited from (a) the horizontal and (b) the vertical port of the OMT.

Therefore, losses in the 8.3-mm waveguide lengths (approximately $0.3 \mathrm{~dB} / \mathrm{mm}$ ) dominate over the small OMT geometry and the widening horn.

In a full transceiver geometry with transmit and receive source chains optimized from the beginning for integration with the OMT duplexer, in contrast to the present situation where the OMT was designed to fit in the pre-existing radar with a grid-based duplexer (Fig. 6), the long WR 1.5 waveguide sections can be substantially shortened with an accompanying reduction in loss. However, reducing the loss through the waveguide sections will also reduce the isolation, leading to the consequences of higher transmit/receive leakage.

Another aspect of the OMT/horn is the cross polarization (XP). In the present case for radar system application, the XP is not a critical parameter unlike in other applications such as communications or radioastronomy receivers. However, it is interesting to obtain an estimation of the XP-based on rigorous full-wave simulations. According to past knowledge [11]-[13], a key parameter responsible of the degradation of the XP in OMT devices is the misalignment errors between split-block. The XP at the common port of the OMT translates directly into the XP level of the radiation pattern at boresight. Moreover, due to the split-block of the horn antenna an additional XP degradation is expected at boresight, as well as at different elevation angles. Fig. 11 shows the simulated radiation patterns (from the vertical and the horizontal ports of the OMT) with a misalignment between blocks of $4 \mu \mathrm{m}$. It can be observed a similar degradation for both polarizations to a level of XP slightly below $-15 \mathrm{~dB}$.

The obtained results compared with the required specifications previously mentioned are summarized in Table I. 
TABLE I

SPECIFICATIONS AND ACHIEVED RESULTS OF THE OMT/HORN

\begin{tabular}{|c|c|c|}
\hline & Specified & Measured \\
\hline Bandwidth & $\begin{array}{c}660-690 \mathrm{GHz} \\
(5 \%)\end{array}$ & $\begin{array}{c}640-710 \mathrm{GHz} \\
(>10 \%)\end{array}$ \\
\hline Return loss & $>15 \mathrm{~dB}$ & $>18 \mathrm{~dB}$ \\
\hline Isolation & $>30 \mathrm{~dB}$ & $>33.5 \mathrm{~dB}$ \\
\hline Insertion loss & $\begin{array}{c}\text { As low as } \\
\text { possible }\end{array}$ & $\begin{array}{c}2.3 \mathrm{~dB} \\
\text { (Mean value) }\end{array}$ \\
\hline $\begin{array}{c}\text { Cross- } \\
\text { polarization }\end{array}$ & Not specified & $\begin{array}{c}<-15 \mathrm{~dB} \text { (full- } \\
\text { wave estimation) }\end{array}$ \\
\hline
\end{tabular}
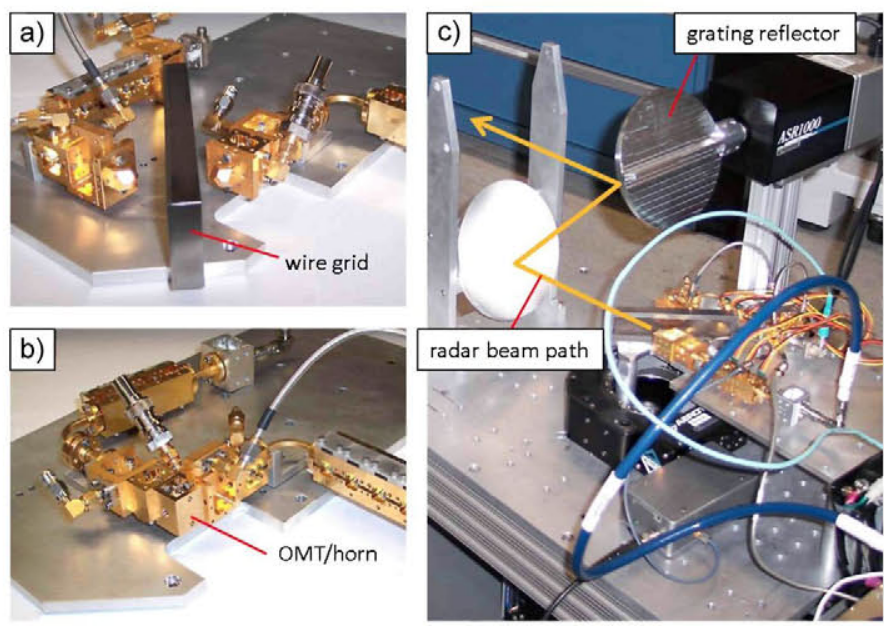

Fig. 12. Photographs of the $680-\mathrm{GHz}$ radar front-end configurations. (a) Wire grid duplexer. (b) OMT/horn duplexer. (c) Front-end showing the grating polarizer and the beam path.

\section{Radar Performance Assessment}

\section{A. Insertion Loss Measurement}

To verify the $680-\mathrm{GHz}$ OMT/horn performance and assess the impact of finite waveguide port isolation, we conducted radar experiments to directly compare the use of the OMT against a wire grid in a circular polarization duplexer. Fig. 12(a) and (b) shows photographs of the two front-end configurations on an aluminum plate that is mounted onto a radar test-bench nearly identical to that reported in [1] and [3]. That radar's subreflector illuminating the main aperture was removed, leaving only a parabolic feed reflector and a flat grating [Fig. 12(c)] to project the collimated beam horizontally across our laboratory. With the OMT horn positioned at the same feed reflector focal point as the transmit horn of the wire grid configuration, this optical system generates a collimated beam with a diameter of $\sim 12 \mathrm{~cm}$ and a diffraction-limited divergence angle of $\sim 0.2^{\circ}$. For the wire grid configuration, diagonal WR 1.5 diagonal horns from Virginia Diodes Inc. were used on the $T x$ and $R x$ ports with a nominal gain of $25 \mathrm{~dB}$, compared with a calculated gain of $25.6 \mathrm{~dB}$ for the integrated horn on the OMT.

For both duplexer configurations, the same radar back-end source and detection electronics were used, based on the architecture described in [1]. An FMCW radar waveform was generated over $662.4-691.2 \mathrm{GHz}$ at a chirp rate of $0.29 \mathrm{GHz} / \mu \mathrm{s}$, so that a target at some standoff range $R$, in meters, appears at an IF frequency of $R \times 1.92 \mathrm{MHz}$. The power generated by the final tripler in the $670 \mathrm{GHz} \times 2 \times 3$ multiplier chain was measured with an Erickson power meter to average $0.9 \mathrm{~mW}$ (or $-0.5 \mathrm{dBm}$ ). The receiver mixer was the same one used to obtain the data in Fig. 10.

The first comparison between the OMT and the wire grid involved measuring the signal-to-noise ratio of a $3.8 \mathrm{~cm}$ diameter aluminum ball mounted in the collimated radar beam positioned at a standoff range of about 2 meters from the flat grating reflector. The aluminum ball was carefully positioned in the center of the beam to maximize the reflected signal energy from the same standoff range for the two transceiver duplexer configurations. Fig. 13(a) shows the results of averaging many radar pulse returns (chirps) until there was negligible random error in the measured power, and plotting the smoothed range-compressed spectra. The ball target at $2.5-\mathrm{m}$ range from the transceiver is clearly visible with an SNR exceeding $30 \mathrm{~dB}$.

We find that the return signal strength when using the wire grid is $4.3 \mathrm{~dB}$ larger than when using the OMT. Gratifyingly, this is very close to the $4.6 \pm 0.2 \mathrm{~dB}$ two-way loss estimate from the Y-factor measurements described in Section III. Note that the return signal from the metal ball is much wider than the bandwidth-limited range resolution of $0.5 \mathrm{~cm}$ because no correction was applied for nonlinearities in the transmitted chirp signal to achieve bandwidth-limited range resolution. The higher noise floor evident in the expanded portion of the spectrum in Fig. 13(a) will be discussed below in Section IV-B.

Two problems with making accurate radar sensitivity measurements using the metal ball target are that systematic errors arise from a variation in the optical system's antenna gain for the two test configurations and that standing wave effects result in a strong sensitivity of the detected signal based on the precise position of the ball target. (Repeated measurements of the same ball target independently aligned by hand to maximize the return signal produce variations in total power detected of about $0.6 \mathrm{~dB}$ ). Therefore as a second measurement of the OMT loss using the radar, we compared the detection signal for the wire grid and the OMT configurations using a large sheet of highly diffusely scattering echosorb as a target. During the few-second measurement period, the echosorb was moved randomly by hand in a plane normal to the beam in the target plane, at a fixed standoff range, to generate signal speckle that could be averaged and quantified accurately. This measurement should be less sensitive to the antenna gain because the target size exceeds the beam size, and it also averages out standing-wave or interference effects. It is also closer to the operational scenario of a radar imaging through diffusely scattering clothing. Fig. 13(b) shows the results of this experiment, where it can be clearly seen that, despite the $>10-\mathrm{dB}$ scatter in detected power for any single measurement, the average signal strength is higher for the wire grid than the OMT. The difference between averaging the powers in decibels of the two measurements is found to be $5.5 \pm 0.1 \mathrm{~dB}$.

It is interesting that estimating the OMT loss from the data of Fig. 13(b) can also be done using the Rayleigh distribution formula for the coherent radar return power $P$ of a scene with random clutter noise [19], $p(P) \sim \exp (-P /\langle P\rangle)$. Taking the natural log of a 15-bin histogram of the power measurements of Fig. 13(b) gives an estimate of the linear equation $\log (p(P))=$ 
a)

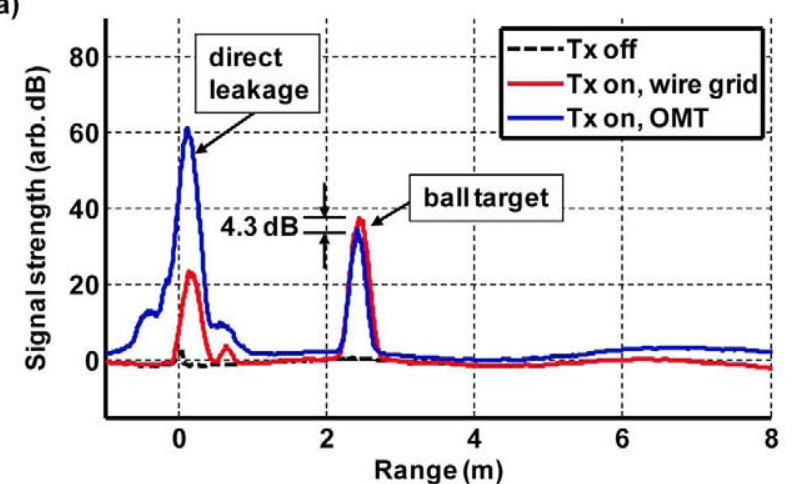

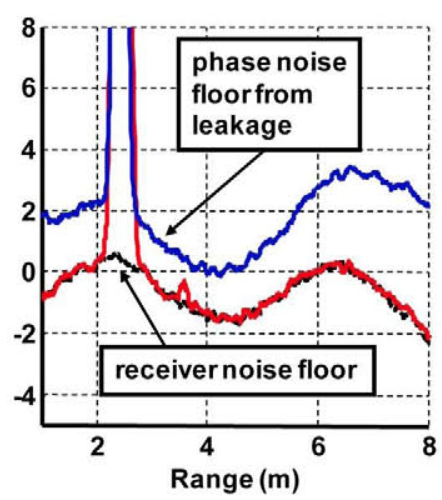

c)

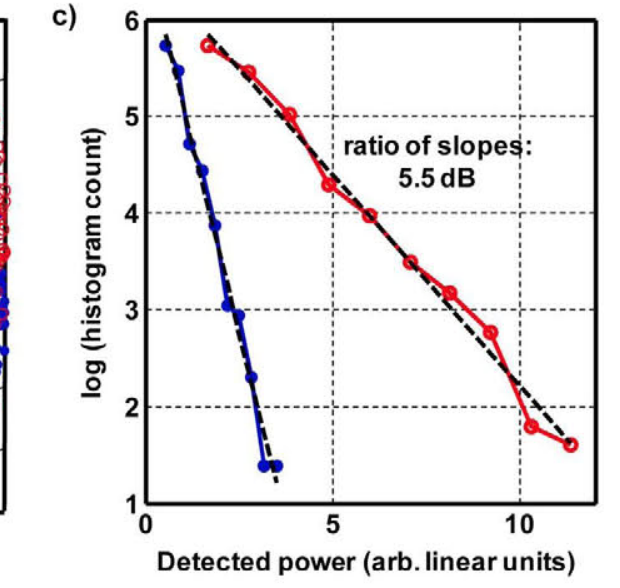

b)

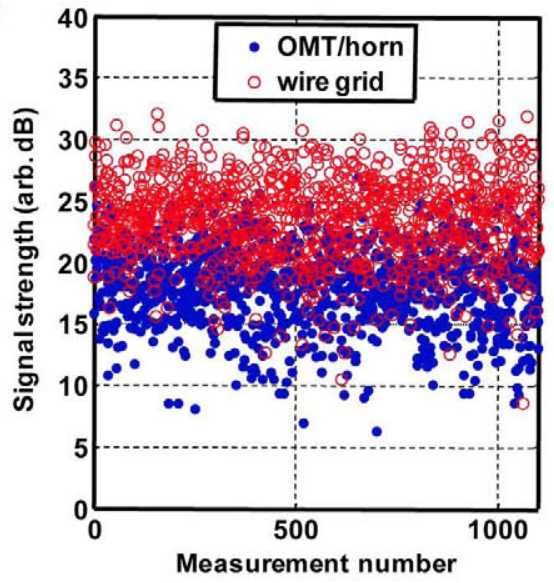

(1)

Fig. 13. Radar performance assessment. (a) Received signal from a 2-m standoff metallic ball target. (b) Received signal from a randomly moving echosorb target. (c) Measured and fitted lines $\log (p(P))=A-P /\langle P\rangle$.

$A-P /\langle P\rangle$ for the two measurements. Then, least-squares fitting of this data with a standard error analysis, where the first (lowest power) point is excluded because it appears to be an outlier of a straight line fit, yields a signal power difference of 5.5 $\pm 0.2 \mathrm{~dB}$, very similar to the simple averaging estimate above but with a larger error. The fitted lines are plotted on top of the measured data in Fig. 13(c), and the good match confirms that the moving-echosorb method of measuring radar power does indeed produce signal statistics consistent with random speckle scattering. Taking the measurements of Fig. 13 together, the measured two-way insertion loss $\left(\left|S_{12}\right|+\left|S_{31}\right|\right)$ for the new OMT is between 4.3 and $5.5 \mathrm{~dB}$, consistent with the Y-factor estimate of $4.6 \mathrm{~dB}$.

\section{B. Noise From Transmit/Receive Leakage}

The $\sim 5$-dB two-way insertion loss is one penalty for using the OMT that must be traded against the potential system advantages of using the component as a more compact and scalable approach to T/R duplexing. This extra loss can likely be significantly reduced by mounting the front-end transmitter and receiver diodes in a single integrated waveguide structure immediately adjacent to the OMT, instead of many millimeters away (as done here; see Fig. 6).

However, reducing the waveguide loss will also worsen the $\mathrm{T} / \mathrm{R}$ isolation, and a close inspection of the magnified plot of Fig. 13(a) reveals that this cannot be tolerated without a corresponding reduction in detection sensitivity. This is because when the transmitter is turned on, even with $33-\mathrm{dB}$ isolation, the receiver noise floor increases above its transmitter-off level by about $1.5-4 \mathrm{~dB}$, depending on the range of the target. The higher noise floor comes from excess noise in the transmitter leaking through the OMT duplexer into the receiver port. This difference would be even larger if a state-of-the-art 670-GHz mixer were used with 10-dB conversion loss [1] instead of the $12-13-\mathrm{dB}$ conversion loss for the mixer used in these experiments, or if a higher power transmitter were used. More detailed analysis and measurement of this phase noise leakage, where it is shown to originate from residual additive phase noise in the submillimeter-wave frequency multiplication chains, is presented in [20].

The thermal noise floor can easily be reached with the transmitter turned on when using the ultrahigh-isolation $(>90 \mathrm{~dB}$ ) quasi-optical wire grid, but not with the OMT's more limited isolation. Thus, the overall penalty for using the OMT in the circular polarization duplexer, with a $\sim 1-\mathrm{mW}$ transmit power and a $\sim 13-\mathrm{dB}$ receiver conversion loss, is roughly $\sim 5 \mathrm{~dB}$ from ohmic loss plus $\sim 3 \mathrm{~dB}$ from excess leakage noise, or $\sim 8 \mathrm{~dB}$ in total. It is imaginable that, in an optimized geometry, the ohmic loss contribution can be reduced by $\sim 3 \mathrm{~dB}$ by reducing the waveguide lengths leading to the OMT. However, this would lower the isolation by a similar amount, leaving the same $\sim 8-\mathrm{dB}$ total sensitivity reduction.

\section{Imaging Radar Quality}

Despite the 8-dB total SNR penalty from using the OMT instead of the wire grid, the quality of through-clothes radar 

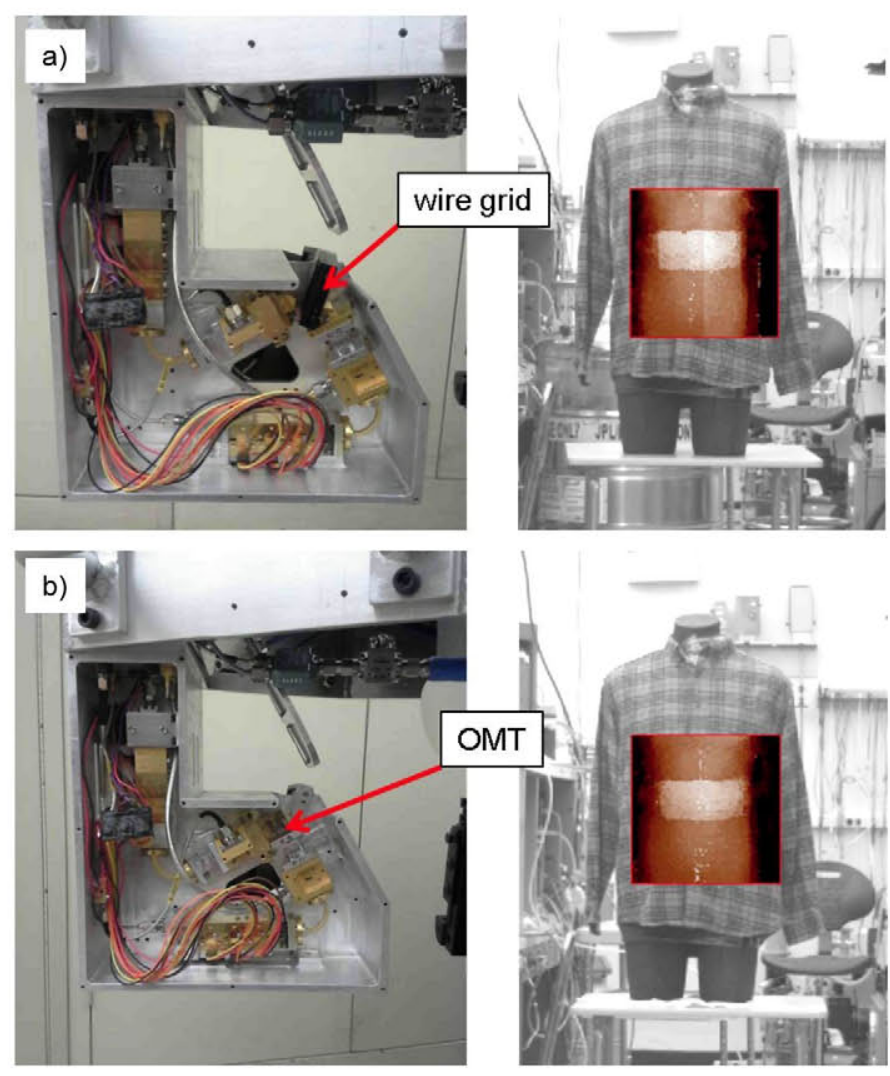

Fig. 14. 680-GHz radar images compared when using (a) a wire grid duplexer and (b) the new OMT duplexer. The image quality for the two transceivers is identical.

images we obtain using our standoff imaging radar system remain unchanged. This can be seen in Fig. 14, which presents radar imagery (following the methods of [1]) of a 1-in-thick mock bomb belt tied to a mannequin's waist and concealed by a flannel cotton shirt. The imaging radar here uses a 50-cm diameter main reflector and operates at a $7-\mathrm{m}$ standoff range. It also scans two beams simultaneously over a $\sim 40-\mathrm{cm}^{2}$ target field of view. These beams are generated in a front-end transceiver enclosure, the right half of which is shown in Fig. 14(a) and (b). For the case of Fig. 14(a), both the left- and the right-hand transceivers are built with the wire grid duplexers. For Fig. 14(b), the new OMT duplexer was used in the right-hand transceiver, as shown in the photograph. However, the $88 \times 88$-pixel radar imagery of the mock bomb belt is essentially identical for the two cases. Thus, as a practical matter, the OMT performance right now can be a suitable substitute for JPL's short-range 680-GHz imaging radar.

\section{CONCLUSION}

In summary, we have designed, built, and thoroughly analyzed the performance of an OMT/horn operating at $680 \mathrm{GHz}$. Very good OMT performance has been demonstrated: return loss greater than $18 \mathrm{~dB}$, isolation more than $33 \mathrm{~dB}$, and insertion loss around $2-3 \mathrm{~dB}$ in a bandwidth exceeding $10 \%$. Ultimately, the measured OMT isolation depends on the $4-\mu \mathrm{m}$ split-block alignment precision.
The OMT/horn has been inserted in the actual 680-GHz radar substituting the wire grid configuration and together with a preexisting grating polarizer constitutes a new robust and compact duplexing alternative, which is especially well suited for submillimeter-wave radar transceivers. The duplexer's performance is excellent, considering the very high frequency of operation. A round-trip power loss of around $5 \mathrm{~dB}$ was measured independently using the radiometric $\mathrm{Y}$-factor method and active radar measurements.

In addition, the short range radar images obtained with the wire grid and the OMT are essentially identical. In this way, the OMT is proved to be a suitable compact solution for the JPL's short-range 680-GHz imaging radar.

\section{ACKNOWLEDGMENT}

The authors would like to thank Prof. C. Groppi and M. Underhill, SESE Terahertz Laboratory, Arizona State University, Tempe, AZ, USA, for the fabrication of the duplexer.

\section{REFERENCES}

[1] K. B. Cooper, R. Dengler, N. Llombart, B. Thomas, G. Chattopadhyay, and P. Siegel, "THz imaging radar for standoff personnel screening," IEEE Trans. THz Sci. Technol., vol. 1, no. 1, pp. 169-182, Sep. 2011.

[2] J. Ding, M. Kahl, O. Loffeld, and P. H. Bolivar, "THz 3-D image formation using SAR techniques: Simulation, processing and experimental results," IEEE Trans. THz Sci. Technol., vol. 3, no. 5, pp. 606-616, Sep. 2013.

[3] K. B. Cooper, N. Llombart, G. Chattopadhyay, B. Dengler, R. E. Cofield, C. Lee, S. Filchenkov, and E. Koposova, "A grating-based circular polarizationduplexer for submillimeter-wave transceivers," IEEE Microw. Wireless Compon. Lett., vol. 22, no. 3, pp. 108-110, Mar. 2012.

[4] C. A. Leal-Sevillano, K. B. Cooper, J. A. Ruiz-Cruz, J. R. MontejoGarai, and J. M. Rebollar, "A $225 \mathrm{GHz}$ circular polarization waveguide duplexer based on a septum orthomode transducer polarizer," IEEE Trans. THz Sci. Technol., vol. 3, no. 5, pp. 574-583, Sep. 2013.

[5] J. Uher, J. Bornemann, and U. Rosenberg, Waveguide Components for Antenna Feed Systems: Theory and CAD. Norwood, MA, USA: Artech House, 1993.

[6] A. M. Bøifot, "Classification of ortho-mode transducers," Eur. Trans. Telecommun., vol. 2, no. 5, pp. 503-510, 1991.

[7] G. Chattopadhyay, B. Philhour, J. Carlstrom, S. Church, A. Lange, and J. Zmuidzinas, "A 96-GHz ortho-mode transducer for the polatron," IEEE Microwa. Guided Wave Lett., vol. 8, no. 12, pp. 421-423, Dec. 1998.

[8] J. M. Rebollar, J. Esteban, and J. de Frutos, "A dual frequency OMT in the Ku band for TTC applications," in Proc. IEEE Antennas Propag. Soc. Int. Symp., Jun. 1998, vol. 4, pp. 2258-2261.

[9] M. Ludovico, B. Piovano, G. Bertin, G. Zarba, L. Accatino, and M. Mongiardo, "CAD and optimization of compact ortho-mode transducers," IEEE Trans. Microw. Theory Techn., vol. 47, no. 12, pp. 2479-2486, Dec. 1999.

[10] J. A. Ruiz-Cruz, J. R. Montejo-Garai, and J. M. Rebollar, "Optimal configurations for integrated antenna feeders with linear dual-polarisation and multiple frequency bands," IET Microw., Antennas Propag., vol. 5, no. 8, pp. 1016-1022, 2011.

[11] C. A. Leal-Sevillano, T. Yingtao, M. J. Lancaster, J. A. Ruiz-Cruz, J. R. Montejo-Garai, and J. M. Rebollar, "A micromachined dual-band orthomode transducer," IEEE Trans. Microw. Theory Techn., vol. 62, no. 1, pp. 55-63, Jan. 2014.

[12] A. Dunning, S. Srikanth, and A. R. Kerr, "A simple orthomode transducer for centimeter to submillimeter wavelengths," in Proc. 20th Int. Symp. Space THz Technol., Charlottesville, VA, USA, 1990, pp. 191-194.

[13] T. J. Reck and G. Chattopadhyay, "A $600 \mathrm{GHz}$ asymmetrical orthogonal mode transducer," IEEE Microw. Guided Wave Lett., vol. 23, no. 11, pp. 569-571, Nov. 2013.

[14] C. Granet, G. L. James, R. Bolton, and G. Moorey, "A smooth-walled spline-profile horn as an alternative to the corrugated horn for wide band millimeter-wave applications," IEEE Trans. Antennas Propag., vol. 52, no. 3, pp. 848-854, Mar. 2004. 
[15] C. Jung-Kubiak, T. J. Reck, J. V. Siles, R. Lin, J. Gill, C. Lee, K. B. Cooper, I. Mehdi, and G. Chattopadhyay, "A multi-step DRIE process to fabricate silicon-based terahertz waveguide components," J. Micromech. Microeng., to be published.

[16] T. J. Reck, C. Jung-Kubiak, J. Gill, and G. Chattopadhyay, "Measurement of silicon micromachined waveguide components at 500 to 750 GHz," IEEE Trans. THz Sci. Technol., vol. 4, no. 1, pp. 33-38, Jan. 2014.

[17] C. A. Leal-Sevillano, T. J. Reck, C. Jung-Kubiak, G. Chattopadhyay, J. A. Ruiz-Cruz, J. R. Montejo-Garai, and J. M. Rebollar, "Silicon micromachined canonical E-plane and H-plane bandpass filters at the terahertz band," IEEE Microw. Guided Wave Lett., vol. 23, no. 6, pp. 288-290, Jun. 2013.

[18] N. Llombart, K. B. Cooper, R. J. Dengler, T. Bryllert, G. Chattopadhyay, and P. H. Siegel, "Time-delay multiplexing of two beams in a terahertz imaging radar," IEEE Trans. Microw. Theory Techn., vol. 58, no. 7, pp. 1999-2007, Jul. 2010.

[19] M. I. Skolnik, "Radar Clutter," in Introduction to Radar Systems, 3rd ed. New York, NY, USA: McGraw-Hill, 2001, pp. 436-437.

[20] K. B. Cooper and R. J. Denger, "Residual phase noise and transmit/receive isolation in a submillimeter-wave FMCW radar," in IEEE Microw. Symp. Dig., Tampa, FL, USA, Jun. 2014, pp. 1-4.

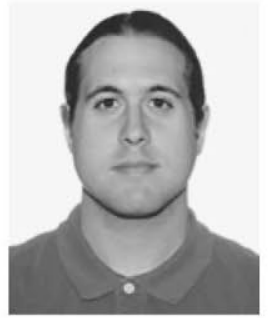

Carlos A. Leal-Sevillano (S'14) was born in Madrid, Spain, in 1986. He received the Telecommunications Engineer degree, M.S. degree, and Ph.D. degree from the Universidad Politécnica de Madrid, Madrid, Spain, in 2009, 2010, and 2014, respectively.

Since 2008 , he has collaborated with the Grupo de Electromagnetismo Aplicado y Microondas at Universidad Politécnica de Madrid. He was a visiting Ph.D. student with the University of Birmingham, Birmingham, U.K., during 2011 and with the NASA Jet Propulsion Laboratory (JPL), California Institute of Technology, Pasadena, CA, USA, during 2012. He was a part-time Lecturer with the Universidad Autónoma de Madrid during 2014 and a Research Fellow with the Universidad Politécnica de Madrid during 2014. His research interests include electromagnetic wave propagation in waveguide structures, analytic and numeric electromagnetic methods, passive devices in the microwave, millimeter-wave and terahertz bands, and optimization techniques.

Dr. Leal-Sevillano was the recipient of the Best Student Paper Award at EuCAP 2014

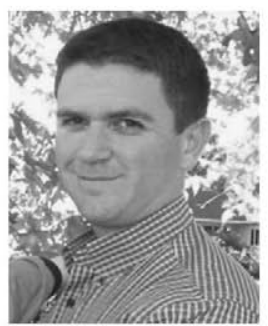

Ken B. Cooper (M'06) received the A.B. degree in physics (summa cum laude) from Harvard College, Cambridge, MA, USA, in 1997, and the Ph.D. degree in physics from the California Institute of Technology, Pasadena, CA, USA, in 2003.

Following postdoctoral research in superconducting microwave devices, he joined the Jet Propulsion Laboratory, Pasadena, CA, USA, as a Member of the Technical Staff in 2006. His current research interests include radar systems up to terahertz frequencies, molecular spectroscopy, and devices.

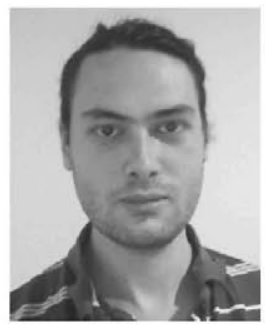

Emmanuel Decrossas (S'08-M'12) received the B.S. and M.S. (with honors) degrees in engineering science and electrical engineering from the Université Pierre et Marie Curie Paris-6, Paris, France, in 2004 and 2006, respectively, and the Ph.D. degree in electrical engineering from the University of Arkansas, Fayetteville, AR, USA, in 2012.

He was a Visiting Scholar Student in 2006 with the University of Tennessee, Knoxville, TN, USA, to initiate an international student exchange program and work on reconfigurable MEMS antennas for wireless applications. He was a Postdoctoral Fellow with the University of Arkansas' High Density Electronics Center (HIDEC) in 2012, where his research involved signal and power integrities signal in LTCC. In December 2012, he joined NASA-Jet Propulsion Laboratory (JPL), Pasadena, CA, USA, after receiving the prestigious NASA postdoctoral fellowship award. His research interests include modeling, optimization, and design of RF/Microwave components, dielectric characterization, computer-aided design of microwave devices, micro-fabrication and nanotechnology to develop and model high-frequency components, and signal and power integrities design.

Dr. Decrossas is member of Eta Kappa Nu. In 2013, he was elected secretary of the IEEE Electromagnetic Compatibility Society technical committee TC-11 on nanotechnology and advanced material.

Robert J. Dengler (M'04) received the B.S. degree in electrical and computer engineering from California State Polytechnic University, Pomona, CA, USA, in 1989.

He began his work at the Jet Propulsion Laboratory, Pasadena, CA, USA, in 1988, developing antenna pattern acquisition and analysis software. Since then, he has been involved with the design and construction of submillimeter-wave receivers and components, including design and fabrication of test instrumentation for submillimeter mixers. He has also worked with terahertz (THz) active and passive imaging systems, including design and construction of a 110-dB dynamic range biosample transmission imaging system at $2.5 \mathrm{THz}$. His recent work is focused on development of a high-resolution THz imaging radar system for contraband detection.

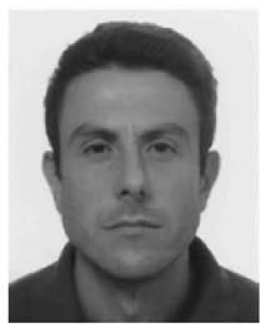

Jorge A. Ruiz-Cruz (SM'11) received the Ingeniero de Telecomunicación degree and Ph.D. degree from the Universidad Politécnica de Madrid, Madrid, Spain, in 1999 and 2005, respectively.

Since 2006, he has been with the Universidad Autónoma de Madrid, Madrid, Spain, where in 2009 he became an Associate Professor. His current research interests are the computer-aided design of microwave passive devices and circuits (e.g., filters, multiplexers, and ortho-modes).

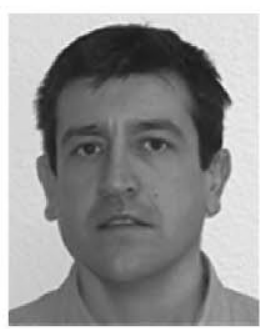

José R. Montejo-Garai was born in Vitoria-Gasteiz, Spain, in 1965 . He received the Ingeniero de Telecomunicación degree and $\mathrm{Ph}$.D. degree from the Universidad Politécnica de Madrid, Madrid, Spain, in 1990 and 1994, respectively.

Since 1989, he has been with the Grupo de Electromagnetismo Aplicado y Microondas at the Universidad Politécnica de Madrid, Madrid, Spain, as an Assistant Professor until 1996, when he was appointed an Associate Professor. His research interests include the analysis and characterization of waveguide structures, advanced synthesis theory, and computer-aided design for microwave and millimeter-wave passive devices: filters, multiplexers, orthomode transducers, and beam-forming networks. He has designed several passive microwave devices for communication satellites.

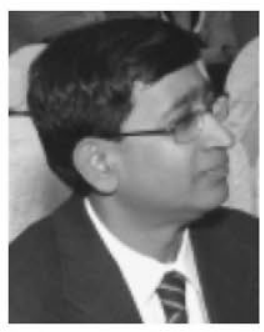

Goutam Chattopadhyay (S'93-M'99-SM'01-F'11) received the $\mathrm{Ph} . \mathrm{D}$. degree in electrical engineering from the California Institute of Technology, Pasadena, CA, USA, in 1999.

He is a Principal Engineer/Scientist with NASA's Jet Propulsion Laboratory, California Institute of Technology, Pasadena, CA, USA, and a Visiting Associate with the Division of Physics, Mathematics, and Astronomy, California Institute of Technology. From 1987 until 1992, he was a Design Engineer with the Tata Institute of Fundamental Research (TIFR), Pune, India. His research interests include microwave, millimeter-, and submillimeter- wave heterodyne and direct detector receivers, frequency sources and mixers in the terahertz region, antennas, SIS mixer technology, direct detector bolometer instruments; InP HEMT amplifiers, mixers, and multipliers; high-frequency radars, and applications of nanotechnology at terahertz frequencies. He has authored and coauthored more than 200 publications in international journals and conferences and holds more than 15 patents. 
Dr. Chattopadhyay was the recipient of the Best Undergraduate Student Award from the University of Calcutta in 1987, the Jawaharlal Nehru Fellowship Award from the Government of India in 1992, and the IEEE Microwave Theory and Techniques Society (MTT-S) Graduate Fellowship Award in 1997. He was the recipient of the Best Journal Paper Award in 2013 by IEEE Transactions on TERAhertz SCIENCE AND TECHNOlogy, and IETE Prof. S. N. Mitra Memorial Award in 2014. He also received more than 30 NASA technical achievement and new technology invention awards. He is an IEEE Distinguished Lecturer.

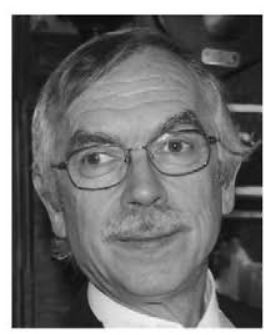

Jesús M. Rebollar was born in Beasain, Spain, in 1953. He received the Ingeniero de Telecomunicación degree and Ph.D. degree from the Universidad Politécnica de Madrid, Madrid, Spain, in 1975 and 1980 , respectively.

Since 1976, he has been with the Grupo de Electromagnetismo Aplicado y Microondas, Universidad Politécnica de Madrid, Madrid, Spain, as an Assistant Professor until 1982 and an Associate Professor until 1988, when he was appointed Professor of Teoría Electromagnética. His research interests include electromagnetic wave propagation in waveguide structures, surface plasmon propagation, interactions of electromagnetic fields with biological tissues, and particularly CAD for microwave and millimeter-wave passive devices: filters, multiplexers, polarizers, orthomode transducers, beam-forming networks, horns, and feeders. He has designed a number of the aforementioned components for communication systems on board satellites. 